TP Periodica Polytechnica Electrical Engineering and Computer Science

60(1), pp. 38-43, 2016

DOI: $10.3311 /$ PPee. 8908

Creative Commons Attribution (1)

RESEARCH ARTICLE

\section{Planar E-Band (71-76 GHz) Platforms for Integrating Millimeter Wave Photodiodes with WR-12 Waveguides}

\author{
Besher Khani $^{1 *}$, Vitaly Rymanov ${ }^{1}$, Sebastian Dülme ${ }^{1}$, \\ Ivan Flammia ${ }^{1}$, Andreas Stöhr ${ }^{1}$
}

Received 25 September 2015; accepted 21 December 2015

\begin{abstract}
A planar 71-76 GHz integration platform is designed and fabricated for high-power radio-over-fiber (RoF) wireless photonic transmitters (PTs). The platform enables the integration of high-electron-mobility-transistor RF amplifiers and millimeter wave photodiodes (PDs), e.g., the waveguide triple transit region $P D$. An efficient electrical coupling between the PD chip and the rectangular waveguide (WR-12) output is attained through a low-loss grounded coplanar waveguide to a rectangular waveguide transition. The integration platform features a novel planar bias-tee design making use of a single quarter-wave coupled-lines (SCLs) section and slotted splitring resonators (SRRS). The bias-tee network enables proper biasing for the PD chip and protects the hybrid integrated RF amplifier from being damaged by the DC bias voltage. The introduced design together with the WR-12 output enables the development of high-power (>17 dBm) PTs for mobile backhaul links and radar applications. Experimental characterization of the designed platform is introduced and compared to numerical results.
\end{abstract}

\section{Keywords}

bias-tee, E-band, photonic transmitter, rectangular waveguide transition, single coupled-lines section, split-ring resonator

\section{Introduction}

The demand to operate in the millimeter wave (mm-wave) frequency bands became inevitable due to the dense usage of the social media and high-definition video broadcasting. In addition to the increased request for mm-wave radar systems employed in security applications, such as personnel imaging [1] and foreign object debris (FOD) [2], it has boomed the wireless communication services [3]. As an enabling technology for such applications, radio-over-fiber (RoF) technology can increase the channel capacity up to multi-gigabit data rates forming fiber-like wireless links [4]. In particular, the recently world-wide allocated E-band frequency slots (e.g., 70/80 GHz band) are considered to be very promising solution for wireless last-mile access and mobile backhauling applications [5], where the atmospheric attenuation has a local minimum relaxing the link budget of wireless networks.

Benefiting from the high carrier frequencies allows the use of simple modulation formats and eliminates the need of digital signal processing in the photonic radio access units, which in its turn simplifies the transmitter architecture, reducing energy consumption and cost. Therefore, broadband photonic transmitters (PTs) with high output power including direct opticalto-wireless conversion have to be developed in order to enable low-cost mm-wave RoF links (Fig. 1).

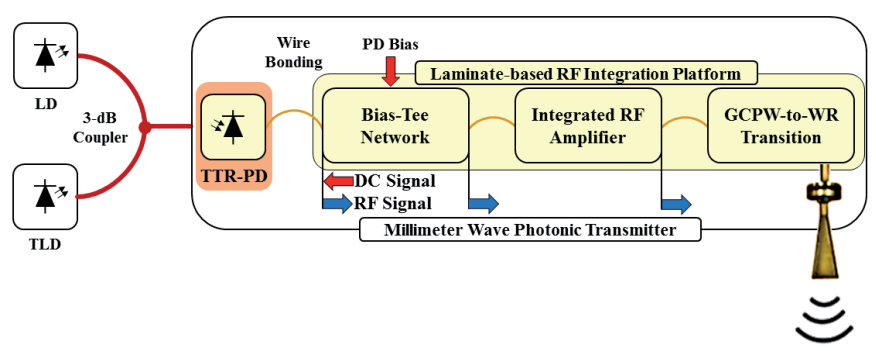

Fig. 1 Concept of the mm-wave wireless photonic transmitter with a rectangular waveguide output.

This article presents the design and fabrication of an E-band (71-76 GHz) integration platform that enables the development of high output power $(>17 \mathrm{dBm})$ PTs for the next generation wireless networks (5G) and mm-wave RoF systems.
${ }^{1}$ Department of Optoelectronics, Faculty of Engineering,

University of Duisburg-Essen, Lotharstr. 55, 47057 Duisburg, Germany

*Corresponding author, e-mail: besher.khani@uni-due.de 


\section{Wireless 71-76 GHz Photonic Transmitter Design}

The concept of a RoF wireless photonic transmitter is presented in Fig. 1. The RF signal generation is realized by optical heterodyning [6]. The optical signals are generated by two laser sources, where the RF frequency can be tuned by varying the wavelength of the tunable laser diode (TLD) while the other laser diode (LD) has a fixed $1550 \mathrm{~nm}$ wavelength. The beat frequency of the optical signals is transmitted over a single-mode fiber (SMF) and subsequently the optical-to-electrical conversion is accomplished by the triple transit region PD (TTR-PD) chip delivering high mm-wave output power $(>0 \mathrm{dBm})$ [7].

Wire-bonding techniques can be used to connect the TTRPD chip having a grounded coplanar waveguide (GCPW) output to a laminate-based GCPW transmission line (TL). The integration platform allows an efficient RF power coupling from the TTR-PD chip to the rectangular waveguide (WR-12), enables proper biasing of the TTR-PD chip, and assists for the $\mathrm{RF}$ amplifier integration in order to design high-power mmwave sources. The designed wireless (71-76 GHz) photonic transmitter is presented in Fig. 2.

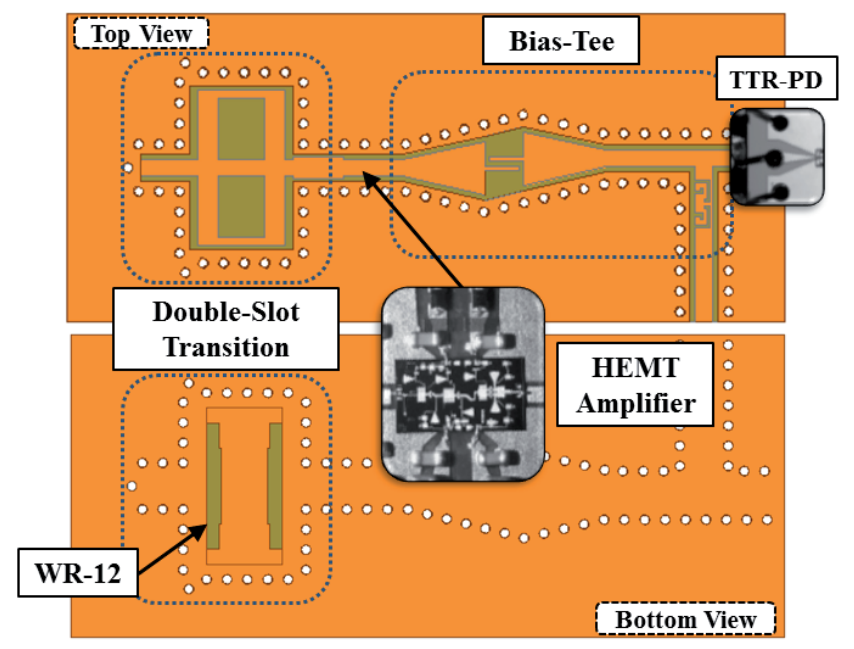

Fig. 2 High-power E-band (71-76 GHz) wireless photonic transmitter design with WR-12 output for rectangular waveguide-fed aperture antennas.

Rectangular waveguides (WRs) play a fundamental role in most high-frequency analogue applications due to their lowloss characteristics. In addition, WRs have often been used as feeds for antennas with sufficient gain to transmit the RF signal to the end-user [9].

The GCPW-based bias-tee (BT) network protects the integrated RF amplifier's input from being damaged by the DC bias voltage by means of a single quarter-wave coupled-lines (SCLs) DC-block. Also, the BT encloses an RF-choke based on a two-slotted split-ring resonators (SRRs) approach in order to prevent the leakage of the RF signal into the DC circuitry.

An RF amplifier integration is required in order to amplify the power delivered by the PD chip. Two RF amplifiers can be integrated in cascade to achieve total gain of $37 \mathrm{~dB}$ (i.e., a low-noise amplifier (LNA) with a $13 \mathrm{~dB}$ gain and a middlepower amplifier (MPA) with a $24 \mathrm{~dB}$ gain). The amplifiers are wire-bonded to the RF integration platform.

The amplified RF signal is coupled out to a rectangular waveguide (WR-12) through a double-slot transition introduced in the bottom ground layer of the laminate [8]. The PT platform is designed on a $127 \mu \mathrm{m}$ thick ROGERS RT/duroid 5880 laminate $\left(\varepsilon_{\mathrm{r}}=2.2\right)$.

\section{Integrated $70 \mathrm{GHz}$ Band Bias-Tee Circuit}

For an efficient biasing of the PD chip, a planar bias-tee circuit is designed and fabricated. It consists of a DC-block and an RF-choke. The design and optimization process are done by means of HFSS ${ }^{(\mathrm{TM})}$ (i.e., a full-wave 3D simulation software based on the finite element method). As previously mentioned, the waveguide TTR-PD chip will be integrated with the laminate-based $50 \Omega$ GCPW integration platform using wire bonding technique.

The DC-block, which is realized using SCLs, blocks the DC signal from running through the transition structure. A bias voltage of $-8 \mathrm{~V}$ is required to properly bias the PD chip to achieve high RF output power levels. In contrast, the RFchoke prevents the loss of the mm-wave signal through the DC circuitry and is based upon two SRRs introduced in the DC bias line with a magnetic coupling distance in between [10]. The integration platform is surrounded by a fence of copper-plated via holes for suppressing higher order modes, which are generated and propagated in the substrate.

\subsection{Single Coupled-Lines Section DC-Block Design}

The SCLs design with tapered impedance matching transition is shown in Fig. 3. The RF coupling occurs between two unshielded TLs, when they are close enough to each other so that interaction of the electromagnetic fields along the lines can take place and the energy from one line is transferred to the neighboring one.

The RF power coupling depends on the physical dimensions of the structure and the input impedance of the SCLs. Tightly-coupled quarter-wave $(\lambda / 4)$ lines with open circuit terminations block the DC signal from running through the platform $[11,12]$. The tapered impedance matching transition is required in order to achieve a smooth impedance transformation from a $50 \Omega \mathrm{GCPW}\left(\mathrm{Z}_{\mathrm{GCPW}}\right)$ to the SCLs' characteristic impedance $\left(Z_{0}\right)$. The mathematical representation, impedance calculations, and design parameters have been introduced in [13]. The inset in Fig. 3 shows a light-microscope close-up view of the fabricated SCLs.

The experimental validation of the fabricated SCLs is introduced in Fig. 4 together with its numerical results within the frequency range of interest from $71 \mathrm{GHz}$ to $76 \mathrm{GHz}$. In order to calibrate and de-embed the scattering parameters (S-parameters) of the cables and connectors used in the experimental 
validation, a dedicated thru-reflect-line (TRL) calibration kit is designed and fabricated (see Fig. 6). The TRL calibration shifts the measurement reference planes up to the device under test (DUT) ports as shown in Fig. 5. The measured results show that the insertion loss (IL: $\mathrm{S}_{21}$ ) is less than $2 \mathrm{~dB}$ in a good agreement compared to the simulation results. The deviation of around 1.5 $\mathrm{dB}$ between the experimental and the numerical results is due to the fabrication tolerance of about $20 \mu \mathrm{m}$ compared to the designed geometry.

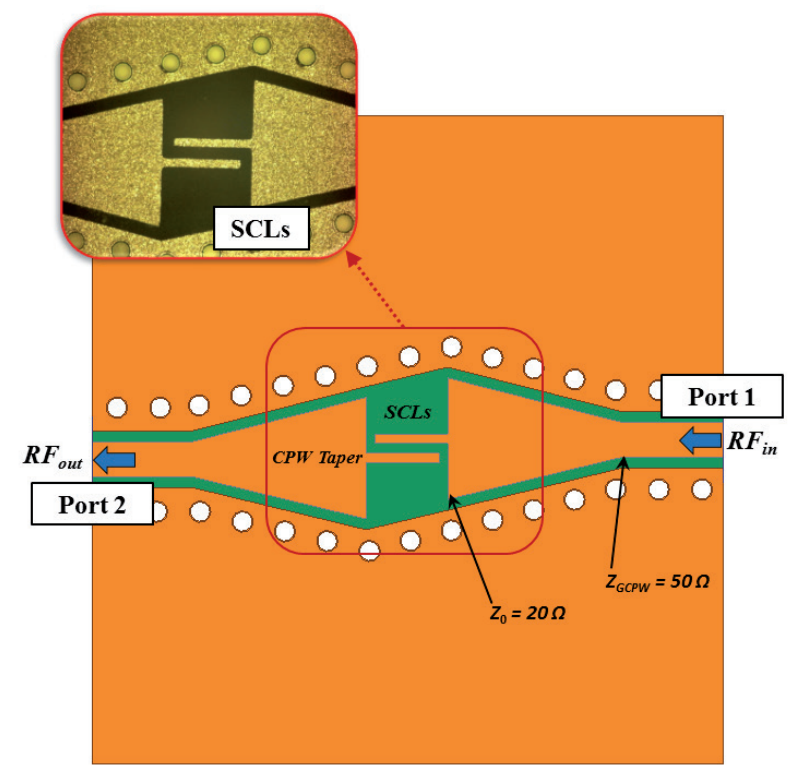

Fig. 3 DC-block: SCLs design with tapered impedance matching transition. The inset shows a microscope close-up view of the fabricated SCLs.

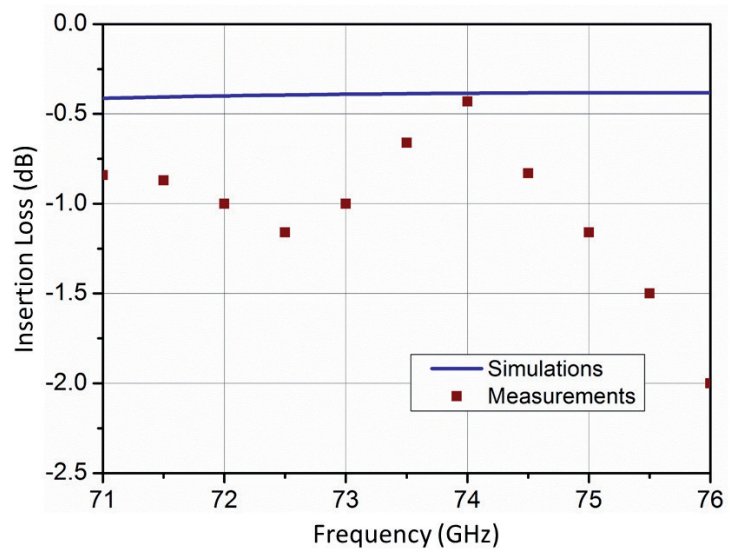

Fig. 4 Insertion loss measurements of the SCLs DC-block compared to the simulated results.

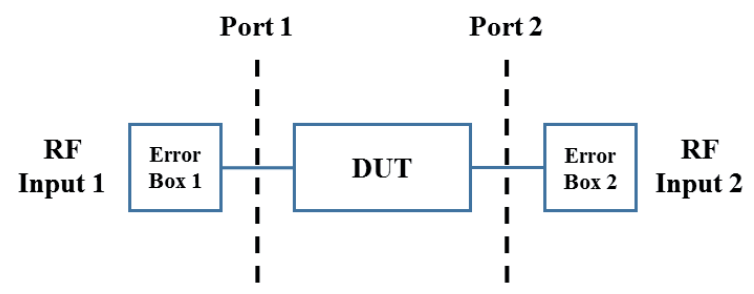

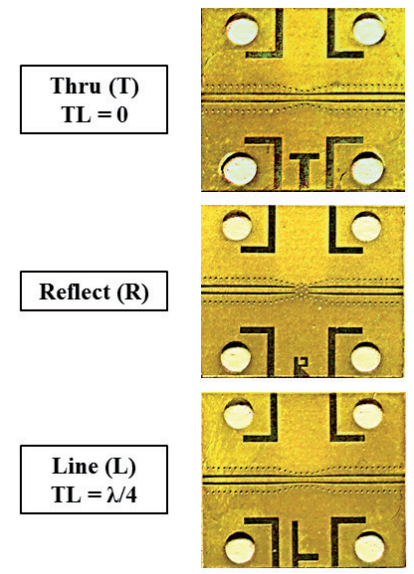

Fig. 6 Thru-reflect-line (TRL) calibration kit (T: zero-length transmission line (TL); R: via holes; L: $\lambda / 4$ length TL).

\subsection{Bias-Tee featuring Split-Ring Resonators RF-Choke Design}

The RF-choke design is based on two slotted SRRs integrated in the DC bias line with a magnetic coupling distance in between. The SRRs design is shown in Fig. 7, together with a microscope view of the fabricated structure (inset). The design approach and the geometrical dimensions of the fabricated SRRs RF-choke are introduced in [10] and [13], respectively. The SRRs' gaps have been set to $60 \mu \mathrm{m}$. The benefit of the SRRs over the quarter-wave based RF-choke reported in [14] is that its length is reduced by $75 \%$ compared to a conventional $\lambda / 4$ approach, which results in a more convenient placing of the RF-choke in the DC circuitry and more compact wireless PT design.

The simulation and optimization of the SRRs structure has been done by HFSS. The calculated and experimental S-parameters are compared and introduced in Fig. 8. The measured RFchoke shows that in the whole $5 \mathrm{GHz}$ bandwidth (BW) the RF signal suppression in the DC bias line (IS: $\mathrm{S}_{31}$ ) is higher than 20 $\mathrm{dB}$ while the IL is lower than $1 \mathrm{~dB}$. The planar bias-tee network from Fig. 9, including both SRRs RF-choke and SCLs DCblock, has further been simulated and fabricated. The insets show the processed parts of the BT circuit. The mm-wave signal is coupled out of the TTR-PD chip (port 1) to the BT output at port 2. The DC bias is applied to the bias-tee at port 3 and supplied to the PD input at port 1 . The simulated and the measured IL $\left(\mathrm{S}_{21}\right)$ results are compared in Fig. 10, showing that the deviation between both is less than $1.5 \mathrm{~dB}$, which is caused by the fabrication tolerances of the processed structures. The measured IL remains below $2 \mathrm{~dB}$ for the complete frequency band of interest.

Fig. 5 Schematic view of the device under test (DUT) measurement calibration. 


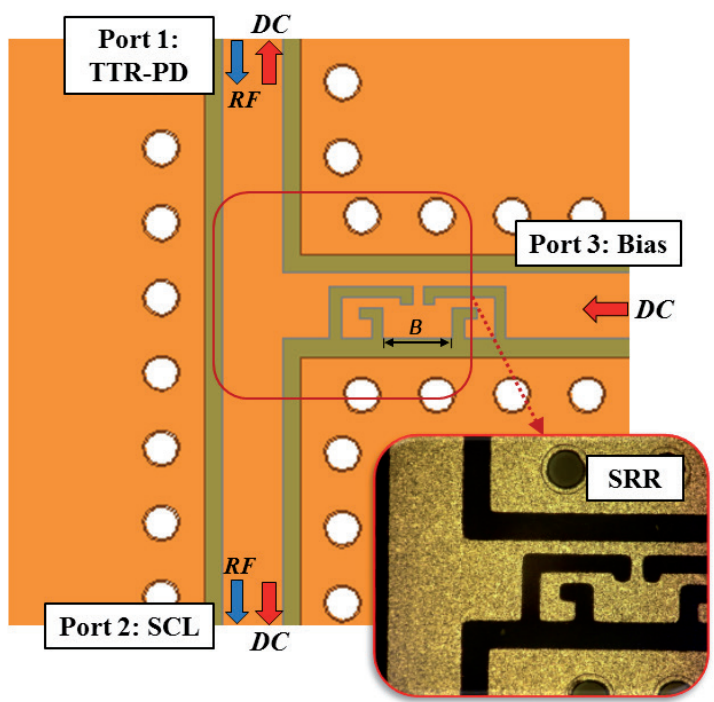

Fig. 7 RF-choke: Two SRRs design with coupling distance in between. The inset shows a microscope close-up view of the fabricated SRRs.

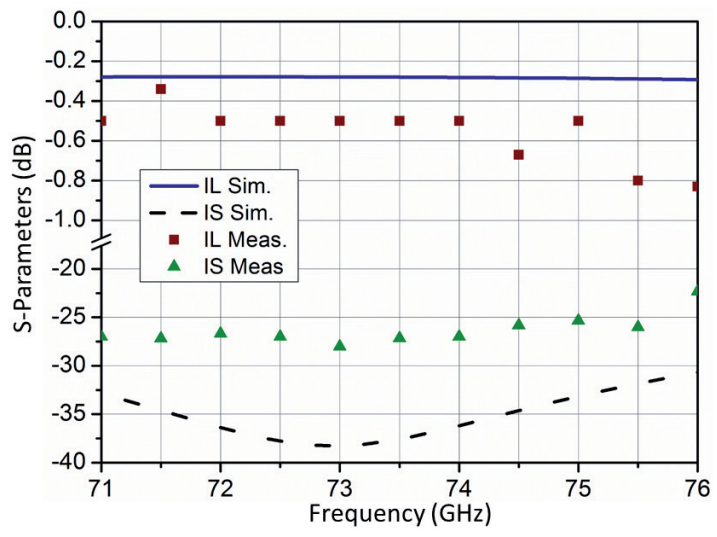

Fig. 8 Insertion loss (IL) and isolation loss (IS) measurements of the SRRs RF-choke compared to the numerical results.

\section{Photonic Transmitter Integration Platform for Radio-over-Fiber Applications}

An easy-to-fabricate $50 \Omega$ GCPW to WR low-loss transition has been designed and characterized in [15] and [16]. The transition has an operational BW from 66-80 GHz. It consists of a two-stage interface, one from the GCPW to the slots, as shown in Fig. 2: (top view), and the other one from the slots to the WR-12 input (Fig. 2: bottom view). The transition makes use of a double-slot antenna coupling approach [8], which guides the electromagnetic field to the WR through the bottom ground layer of the laminate.

The 71-76 GHz integration platform design with the biastee circuit and the double-slot transition based on ROGERS RT/duroid 5880 substrate is shown in Fig. 11. The designed integration platform allows an efficient coupling of the RF signal, which is provided by a high-speed PD chip (e.g., TTRPD [17]), to the WR-12 output. In addition, it allows further RF power amplification by integrated RF amplifiers, which are integrated by means of bond wires to the laminate-based
GCPW between the double-slot transition and the DC-block. Fig. 11a shows the propagation of the electromagnetic field at $73.6 \mathrm{GHz}$ in the dielectric substrate of the integration platform and the coupling of the electromagnetic waves to the WR-12 (Fig. 11b). It can be noticed that no RF power is observed in the DC circuitry. The fabricated PT integration platform is presented in Fig. 11c. The measurement of the platform presented in Fig. 12 shows that the IL is less than $5 \mathrm{~dB}$ for the entire $\mathrm{BW}$, along with a maximum $2.5 \mathrm{~dB}$ deviation, compared to the simulated results due to the fabrication tolerance of about 20 $\mu \mathrm{m}$ occurring in all sections of the platform.

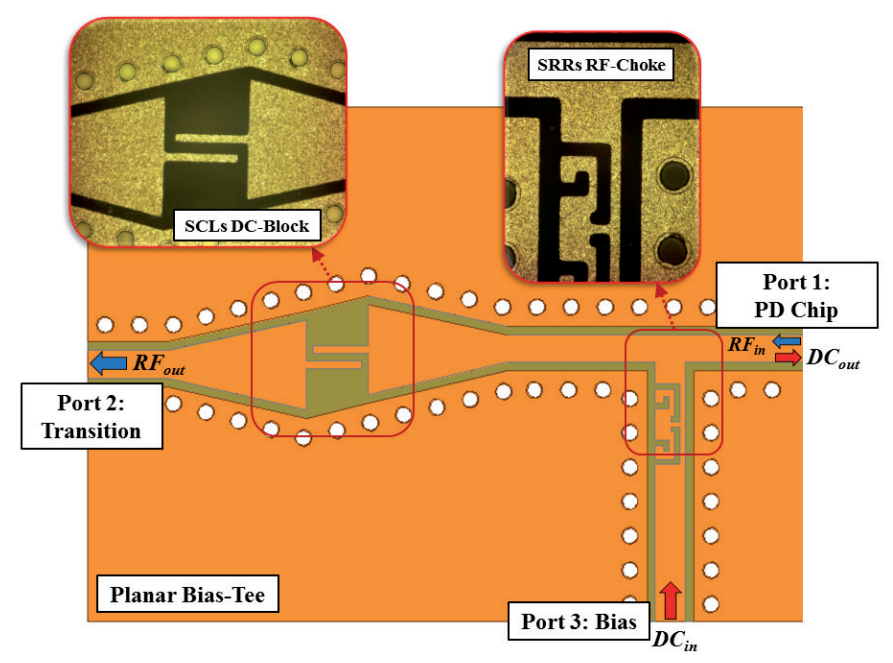

Fig. 9 Planar 71-76 GHz bias-tee circuit design showing the fabricated RF-choke and DC-block in the right and the left inset, respectively.

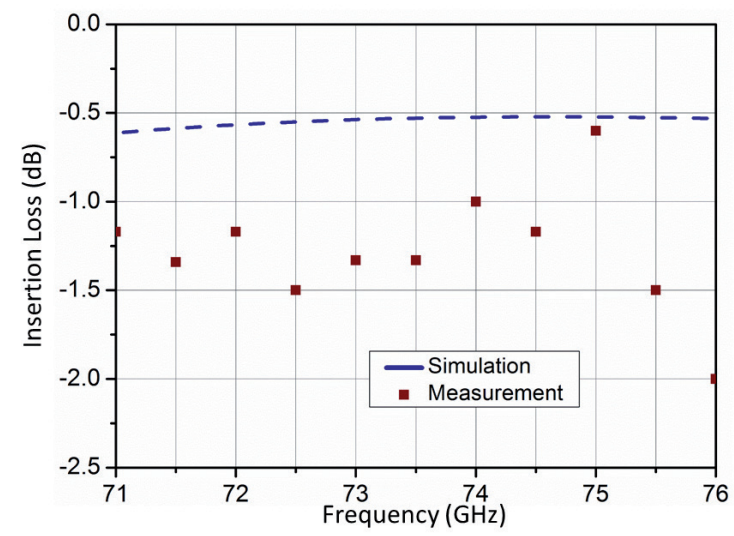

Fig. 10 Comparision of the measured and simulated insertion loss of the planar bias-tee circuit.

\section{Conclusion}

A laminate-based 71-76 GHz RF integration platform for next generation wireless networks $(5 \mathrm{G})$ and radar applications has been designed, fabricated, and experimentally characterized. The platform allows low-cost development of high-power radio-over-fiber wireless photonic transmitters. Experimentally, the integration platform provides a low insertion loss of around $4.5 \mathrm{~dB}$ in the whole $5 \mathrm{GHz}$ bandwidth. Experimental 


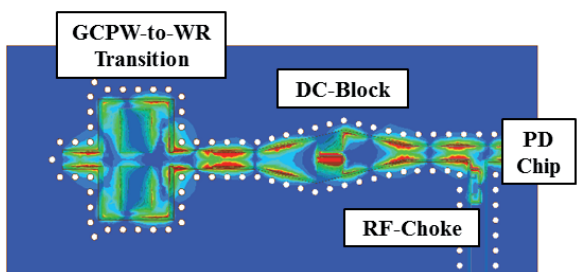

(a)
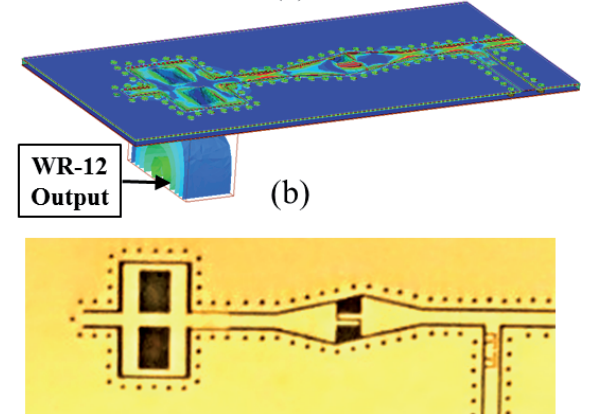

(c)

Fig. 11 Planar integration platform design for wireless $(71-76 \mathrm{GHz})$ photonic transmitter: (a) Top view, (b) 3D view with electric field distribution at $73.6 \mathrm{GHz}$, and (c) the fabricated laminate.

results showed a good agreement with the numerical results. Observed max. $2.5 \mathrm{~dB}$ deviation to the simulated results was traced back to the fabrication tolerances of approx. $20 \mu \mathrm{m}$.

\section{Acknowledgement}

The work presented in this article is supported by the European STReP IPHOBAC-NG (www.iphobac-ng.eu) under the grant no. 619870 .

\section{References}

[1] Ahmed, S. S., Schiessl, A., Schmidt, L. "A novel fully electronic active real-time imager based on a planar multistatic sparse array." IEEE Transactions on Microwave Theory and Techniques. 59 (12). pp. 3567-3576. 2011. DOI: 10.1109/TMTT.2011.2172812

[2] Kohmura, A., Futatsumori, S., Yonemoto, N., Okada, K. "Optical fiber connected millimeter-wave radar for FOD detection on runway." In: $E u$ ropean Radar Conference, EuRAD, pp. 41-44. Oct. 9-11, 2013. URL: $\mathrm{http}$ ://ieeexplore.ieee.org/stamp/stamp.jsp?tp=\&arnumber $=6689108 \&$ is number $=6689067$

[3] Stöhr, A., Babiel, S., Cannard, P. J., Charbonnier, B., Van-Dijk, F., Fedderwitz, S., Moodie, D., Pavlovic, L. L., Ponnampalam, L., Renaud, C. C., Rogers, D., Rymanov, V., Seeds, A., Steffan, A. G., Umbach, A., Weiss, M. "Millimeter-wave photonic components for broadband wireless systems." IEEE Transactions on Microwave Theory and Techniques. 58 (11). pp. 3071-3082. 2010. DOI: 10.1109/TMTT.2010.2077470

[4] Seeds, A. J., Shams, H., Fice, M. J., Renaud, C. C. "Tera Hertz photonics for wireless communications." Journal of Lightwave Technology. 33 (3). pp. 579-587. 2015. DOI: 10.1109/JLT.2014.2355137

[5] Stöhr, A., Cojucari, O., van Dijk, F., Carpintero, G., Tekin, T., Formont, S., Flammia, I., Rymanov, V., Khani, B., Chuenchom, R. "Robust 71-76 $\mathrm{GHz}$ radio-over-fiber wireless link with high-dynamic range photonic assisted transmitter and laser phase-noise insensitive SBD receiver." In: Optical Fiber Communications Conference and Exhibition, OFC, pp. 1-3, 9-13 Mar. 2014. DOI: 10.1364/OFC.2014.M2D.4

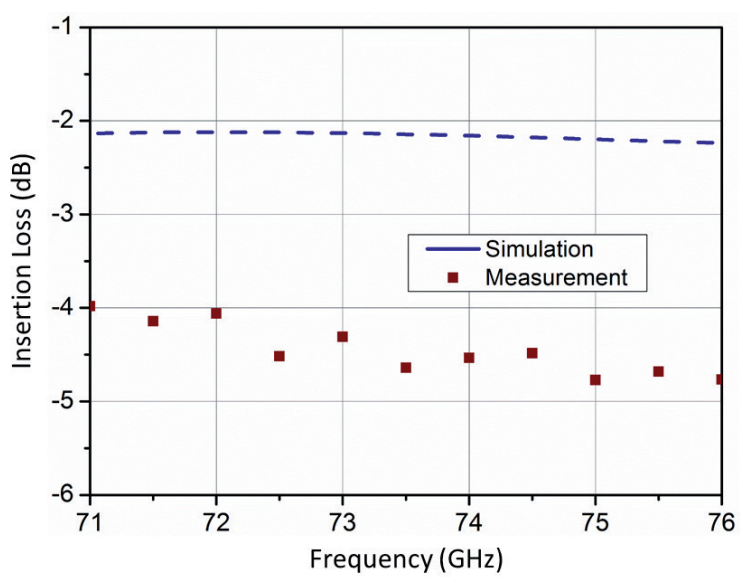

Fig. 12 Insertion loss measurement of the fabricated $71-76 \mathrm{GHz}$ integration platform compared to the simulation results.
[6] Preu, S., Döhler, G. H., Malzer, S., Stöhr, A., Rymanov, V., Göbel, T., Brown, E. R., Feiginov, M., Gonzalo, R., Beruete, M., Navarro-Cía, M. "Principles of THz generation." In: Carpintero, G., García Muñoz, L. E., Hartnagel, H. L., Preu, S., Räisänen, A. V. (eds.) Semiconductor Terahertz Technology: Devices and Systems at Room Temperature Operation. pp. 3-68. Chichester: John Wiley \& Sons, Ltd. 2015.

DOI: 10.1002/9781118920411.ch2

[7] Rymanov, V., Stöhr, A., Dülme, S., Tekin, T. "Triple transit region photodiodes (TTR-PDs) providing high millimeter wave output power." $\mathrm{Op}$ tics Express. 22 (7). pp. 7550-7558. 2014. DOI: 10.1364/OE.22.007550

[8] Radiom, S., Aliakbarian, H., Reynaert, P., Vandenbosch, G., Gielen, G. "A fully micromachined W-band waveguide-to-grounded coplanar waveguide transition for 91-113 GHz applications." In: European Microwave Conference, EuMC, pp. 668-670, 28-30 Sept. 2010. URL: http://ieeexplore.ieee. org/stamp/stamp.jsp?tp=\&arnumber=5616220\&isnumber $=5614756$

[9] Rymanov, V., Khani, B., Dülme, S., Steeg, M., Stöhr, A. "Compact triple transit region photodiode module with WR-12 rectangular waveguide output." In: IEEE International Topical Meeting on Microwave Photonics, MWP, pp. 1-4, 26-29 Oct. 2015. DOI: 10.1109/mwp.2015.7356701

[10] Khani, B., Rymanov, V., Steeg, M., Buck, A., Dülme, S., Stöhr, A. "Compact E-Band (71-86 GHz) Bias-Tee Module for External Biasing of Millimeter Wave Photodiodes." In: IEEE International Topical Meeting on Microwave Photonics. MWP, pp. 1-4, 26-29 Oct. 2015. DOI: 10.1109/mwp.2015.7356673

[11] Mongia, R. K., Bahl, I. J., Bhartia, P., Hong, J. "Coupled-line circuit components." In: RF and Microwave Coupled-Line Circuits. pp. 443479. Artech House, Norwood, MA. 2007.

[12] Flammia, I., Khani, B., Stöhr, A. "A novel transition from grounded coplanar waveguide to substrate integrated waveguide for $60 \mathrm{GHz}$ radioover-fiber photonic transmitters." In: Conference on Microwave Techniques, COMITE, pp. 73-76, 17-18 Apr. 2013.

DOI: 10.1109/COMITE.2013.6545046

[13] Khani, B., Rymanov, V., Flammia, I., Miech, M., Stöhr, A. "Planar biastee circuit using single coupled-line approach for 71-76 GHz photonic transmitters." In: German Microwave Conference, GeMiC, pp. 276-279, 16-18 Mar. 2015. DOI: 10.1109/GEMIC.2015.7107807 
[14] Bouhlal, B., Lutzmann, S., Palandöken, M., Rymanov, V., Stöhr, A., Tekin, T. "Integration platform for $72 \mathrm{GHz}$ photodiode-based wireless transmitter." In: SPIE Photonics West, pp. 8259-82590H, 21-26 Jan. 2012. DOI: $10.1117 / 12.909726$

[15] Khani, B., Rymanov, V., Flammia, I., Stohr, A. "Planar 71-76 GHz laminate integration platform for connecting millimeter wave photodiodes to WR-12 waveguides." In: International Conference on Transparent Optical Networks, ICTON, pp. 1-4, 5-9 Jul. 2015

DOI: 10.1109/ICTON.2015.7193499

[16] Flammia, I., Stöhr, A., Leonhardt, C. C., Honecker, J., Steffan, A. G. "71-76 GHz grounded CPW to WR-12 transition for quasi-hermetic RoF wireless transmitter." Electronics Letters. 48 (9). pp. 506-508. 2012. DOI: 10.1049/el.2012.0377

[17] Rymanov, V., Khani, B., Dülme, S., Lu, P., Stöhr, A. "InP-based waveguide triple transit region photodiodes for hybrid integration with passive optical silica waveguides." Photonics. 2 (4). pp. 1152-1163. 2015. DOI: $10.3390 /$ photonics 2041152 